\title{
Podostemum rutifolium subsp. rutifolium como estruturador da comunidade de algas perifíticas em um rio neotropical
}

\author{
Podostemum rutifolium subsp. rutifolium as structuring plant of periphytic algal community
}

in a neotropical river

\author{
Fernando Alves Ferreira ${ }^{1}$, Roger Paulo Mormul ${ }^{1}$, Stefania Biolo ${ }^{1}$ \& Liliana Rodrigues ${ }^{1}$
}

\begin{abstract}
Resumo
As plantas da família Podostemaceae possuem caráter riacófilo, crescendo sobre substrato rochoso. O presente estudo foi desenvolvido no rio Paraná com o objetivo de registrar Podostemum rutifolium Warm. subsp. rutifolium e analisar a composição, a riqueza e a densidade da comunidade de algas perifiticas nesta macrófita. Para amostragem, foi utilizado um quadrado de borracha $\left(25 \mathrm{~cm}^{2}\right)$, mergulho em apnéia e a remoção da planta aderida à rocha. Posteriormente foi realizada a remoção, fixação, identificação e contagem das algas perifiticas deste substrato. Foram identificadas 137 espécies de algas perifíticas distribuídas em nove classes. O maior número de táxons pertenceu à classe Bacillariophyceae, seguida por Cyanophyceae. Esse padrão já foi encontrado para a maioria dos ambientes lóticos da planície de inundação do rio Paraná. Embora seja uma espécie de hábito incrustante, a arquitetura de $P$. rutifolium fornece microhabitats para muitas espécies de algas. Portanto, sugere-se que essa macrófita seja uma espécie chave na estruturação da comunidade algal perifítica e sua remoção poderia acarretar no declínio na diversidade do perifiton, principalmente por ser a única espécie presente como substrato vegetal na área amostrada.
\end{abstract}

Palavras-chave: colonização, estrutura de habitat, água corrente.

\begin{abstract}
Plants from the family Podostemaceae grow on rocks in running water. This study was done in the Paraná River aiming to record the occurrence of Podostemum rutifolium Warm. subsp. rutifolium and to analyze composition, richness and density of the periphytic algae community on this macrophyte. We used a square rubber sampler $\left(25 \mathrm{~cm}^{2}\right)$ and dives in apnea to remove plants attached to the rocks using a putty knife. Later we proceeded to remove and fix, identify and count the algae attached to this substrate. One hundred thirtyseven species of algae were identified and included in nine classes. The highest number of taxa was recorded for Bacillariophyceae, followed by Cyanophyceae. This pattern is found in the majority of lotic environments from the Paraná River floodplain. In spite of being a fouling species, the architecture of $P$. rutifolium provides microhabitats for many algal species. We strongly suggest that this macrophyte is a key species in periphytic algal community structure, whereas its removal could lead to a decline in periphyton diversity mainly due to the unique presence of $P$. rutifolium as plant substrate in the sampling area.
\end{abstract}

Key-words: Colonization, habitat structure, running water.

\section{Introdução}

O conceito "keystone structure" têm sido bem aplicado à presença de uma espécie estruturadora, que por sua vez afeta a diversidade dos grupos associados ou aderidos a ela (Paine 1969; Mills et al. 1993). Este conceito pode estar diretamente relacionado com a estruturação dos habitats aquáticos por macrófitas, principalmente, quando houver uma única espécie como substrato vegetal no ambiente (Estes et al. 2011). A remoção dessa espécie estruturadora pode levar ao declínio da diversidade dos demais grupos, acarretando uma reação em cadeia, destacando-se a vulnerabilidade das comunidades (Tews et al. 2004).

'Universidade Estadual de Maringá - UEM, Pós-graduação em Ecologia de Ambientes Aquáticos Continentais, PEA, Núcleo de Pesquisas em Limnologia, Ictiologia e Aquicultura, Nupelia, Av Colombo 5790, bl. H90, 87020-900, Maringá ,PR. 
Nos ecossistemas aquáticos, as zonas litorâneas são consideradas áreas com alta biodiversidade e um dos fatores que, em geral, está associado a esse fato é a presença de plantas aquáticas (Esteves 1998; Thomaz et al. 1997). Além da estruturação física, as plantas aquáticas proporcionam o aumento da complexidade espacial, o que afeta diretamente a diversidade de espécies associadas a essa vegetação (Thomaz et al. 1997; Wetzel 2001; Murphy et al. 2003; Agostinho et al. 2007). As macrófitas oferecem um excelente substrato para o desenvolvimento, por exemplo, da comunidade perifítica (Rodrigues et al. 2003).

Na planície de inundação do alto rio Paraná, alguns estudos revelam que a diversidade de peixes e de algas perifíticas está fortemente relacionada à diversidade de macrófitas tanto em pequena, como em grande escala (Agostinho et al. 2003, 2007; Murakami et al. 2009). Isto porque a presença de muitas espécies de macrófitas, como matéria orgânica viva ou morta, e do próprio perifíton presente nestas plantas podem servir como fonte de alimento para organismos aquáticos e terrestres (Campeau et al. 1994; Jones et al. 2000; Lopes et al. 2007; Mormul et al. 2010). A partir disso, as macrófitas juntamente ao perifíton, regulam os fluxos energéticos dos sistemas aquáticos como um todo, contribuindo também com elevadas taxas de produtividade nesse sistema (Rodrigues et al. 2003).

A planície de inundação do alto rio Paraná constitui o último trecho deste rio em território brasileiro, o qual consiste num ecossistema tipo rio-planície de inundação (sensu Neiff 1990). Esse trecho possui grande variabilidade de habitats, conservando assim uma elevada diversidade de espécies. $\mathrm{O}$ principal fator que regula a estrutura e o funcionamento das comunidades nesse ecossistema é o pulso de inundação (Thomaz et al. 2007).

Um largo levantamento de espécies aquáticas vem sendo desenvolvido na planície de inundação do alto rio Paraná desde o ano 2000 (Thomaz et al. 2007; Ferreira et al. 2011). Antes do programa de Pesquisas Ecológicas de Longa Duração - PELD, havia um registro de cerca de 60 espécies de plantas aquáticas (Thomaz et al. 1997). Contudo, ao longo das pesquisas esse número elevou-se para 155 (Ferreira et al. 2011). Dentre essas espécies, um representante da família Podostemaceae, Podostemum rutifolium Warm. subsp. rutifolium, merece destaque, pois trata-se de um dos táxons de ocorrência mais comum da família Podostemaceae para o Sul do Brasil, o qual para a
Bacia do Paraná, especificamente, Philbrick et al. (2010) registraram oito espécies. Além da Bacia do Paraná, estes mesmos autores verificaram uma grande e diferenciada flora de Podostemaceae especialmente para o Brasil, em grandes regiões hidrográficas (Bacia do rio Amazonas) e grandes áreas (região sudeste). De uma forma ampla, esta família encontra-se distribuída predominante na faixa tropical, desde o México até a Argentina central além do continente africano central, sul e sudeste da Ásia e norte da Austrália e Nova Guiné (Cook 1990).

As plantas da família Podostemaceae são caracterizadas pela estrutura submersa, pelo hábito incrustante em substratos rochosos e pelo desenvolvimento em águas correntes turbulentas (Cook 1990; Philbrick et al. 2010). Quando se trata de $P$. rutifolium - apesar de ter sido registrada na região limnética de ambiente lêntico, sob influência do movimento de ondas na região (Irgang et al. 2003), esta espécie é tipicamente riacófila. Considerações ecológicas direcionadas à esta espécie ainda são escassas, bem como demais estudos acerca de sua biologia (Philbrick et al. 2010). A importância ecológica da presença de Podostemaceae já foi foco de trabalhos com cadeias alimentares, as quais tanto a própria planta como os organismos aderidos a ela serviram como recurso alimentar para peixes "lambaris" (gênero Astyanax Baird \& Girard) (Vilella et al. 2002).

Nas últimas décadas, vem sendo registrado o aumento dos impactos ambientais antrópicos no alto Rio Paraná e diversas espécies acabaram extintas, local ou globalmente (Maltchik \& Callisto 2004). O desenvolvimento de levantamentos ou inventários tornou-se uma ferramenta fundamental para a quantificação da riqueza de espécies, servindo como apoio para avaliação de toda a biodiversidade. Ainda, atua na detecção da presença de espécies raras e de potenciais espécies invasoras ou exóticas que possam causar danos ao ecossistema. Detalhes oriundos da comunidade perifítica, principalmente seu componente algal, permanece ainda desconhecidos para $P$. rutifolium como um tipo de substrato. Para a planície de inundação do alto rio Paraná, outras macrófitas foram alvo de estudos acerca da comunidade algal aderida, as quais constam: Eichhornia azurea Kunth (Rodrigues \& Bicudo 2001b; Algarte et al. 2006; Murakami et al. 2009), Ricciocarpus natans (L.) Corda (Biolo \& Rodrigues 2010), Nymphaea amazonum Martius \& Zucarini (Rodrigues et al. 2008), Hydrilla verticillata (L.) Royle, Cabomba furcata Schultes \& Schultes e Egeria najas Planch. (Mormul et al. 2010). 
A maioria dos habitats aquáticos da planície de inundação do alto rio Paraná pode ser caracterizada como lêntico ou semilótico, com margens hidromórficas que suportam elevada diversidade de macrófitas aquáticas. Por outro lado, o rio Paraná possui elevado fluxo de água e margens arenosas, suportando menor diversidade de macrófitas, principalmente por essas características impedirem a permanência de plantas flutuantes e a fixação de macrófitas anfíbias e emergentes, que são os três grupos mais ricos em espécie na região (Ferreira et al. 2011). Buscou-se, desta maneira, com o presente trabalho, complementar os registros de ocorrência de Podostemum rutifolium para o estado do Paraná, especificamente para a planície de inundação do alto rio Paraná; e, primariamente, conhecer alguns detalhes acerca da comunidade de algas perifíticas nesta macrófita, a partir da análise da composição, riqueza e densidade algal, tendo em vista ser essa espécie de macrófita a única incrustante encontrada na área de estudo.

\section{Material e Métodos}

A área amostrada encontra-se cerca de $100 \mathrm{~m}$ à montante da base de pesquisas do Núcleo de Pesquisas em Limnologia, Ictiologia e Aquicultura - Nupélia, no município de Porto Rico-PR, na margem esquerda do rio Paraná, na planície de inundação do alto rio Paraná (entre 22 45 ' 52,94 'S e

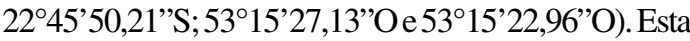
planície é parte integrante da Área de Proteção Ambiental das Ilhas e Várzeas do Rio Paraná, a qual possui área de 1.000.310 ha. Dentro dessa área de proteção estão dois parques, o Parque Nacional de Ilha Grande e o Parque Estadual do Ivinhema que inclui a área de várzea mais importante da região, porque não representa um trecho represado (Agostinho et al. 1994).

Os exemplares coletados de $P$. rutifolium subsp. rutifolium (Fig. 1), em fevereiro de 2009 (águas altas), encontravam-se aderidos às rochas submersas do tipo arenito. Através de mergulho em apnéia, um quadrado fixo de $25 \mathrm{~cm}^{2}$ foi colocado em contato com a rocha. Rapidamente, o interior do quadrado foi removido com auxilio de uma espátula de mesma área. Imediatamente, o material foi prensado entre a espátula e um pequeno pedaço de plástico para reduzir perdas de material aderido, uma vez que esse procedimento teve que ser realizado submerso. Em seguida, as amostras foram acondicionadas em frascos de vidro e mantidas em uma caixa de isopor com gelo (aproximadamente $10^{\circ} \mathrm{C}$ ). Além disso, foi registrada a velocidade da água $\left(\mathrm{m} . \mathrm{s}^{-1}\right)$ com um fluxímetro e verificada a transparência (profundidade) com auxílio do disco de Secchi (m).

Plantas de três profundidades $(0,3,1,5$ e $2,3 \mathrm{~m}$, daqui em diante $\mathrm{P} 1$, subsuperfície; $\mathrm{P} 2$, região intermediária; e P3, região profunda, respectivamente) foram amostradas em réplicas $(n=2)$. Em laboratório, o material perifítico foi removido das plantas com auxílio de pincel de cerdas macias e jatos de água destilada, imediatamente fixado em solução de Transeau na proporção 1:1 para a análise qualitativa, enquanto que para análise quantitativa as amostras foram fixadas em Lugol acético 5\% (Bicudo \& Menezes 2006). As amostras de material perifítico encontram-se depositadas no Herbário da Universidade Estadual de Maringá (HUEM) sob a numeração 20110 a 20115, enquanto os espécimes de $P$. rutifolium subsp. rutifolium estão depositados com o número de tombo 20826.

A análise qualitativa da comunidade de algas procedeu-se por meio da preparação de lâminas temporárias em microscopia óptica com ocular micrometrada. Os táxons encontrados foram medidos e identificados com base na bibliografia clássica (Prescott et al. 1981; Förster 1969; Prescott 1982; Komárek \& Fott 1983; Komárek \& Anagnostidis 1986, 1999, 2005; Krammer \& LangeBertalot 1986, 1988, 1991a,b; Anagnostidis \& Komárek 1988) e regional. A análise quantitativa foi realizada em microscópio invertido com o uso de câmaras de sedimentação, seguindo o método de Utermöhl (1958) e através de campos aleatórios, conforme recomendação de Bicudo (1990). A equação para o cálculo da densidade seguiu Ros (1979), adaptada para a área do substrato, sendo que os resultados foram expressos por unidade de área (ind. $\mathrm{cm}^{-2}$ ).

\section{Resultados}

Dentre as profundidades aqui amostradas no rio Paraná, registrou-se diferenças nos valores de velocidade da água, sendo que da superfície para o fundo a correnteza estava a 2,7; 1,5 e 1,6 m.s. respectivamente. A profundidade do disco de Secchi foi total $(3,5 \mathrm{~m})$, denotando grande transparência no ponto amostrado. A macrófita $P$. rutifolium subsp. rutifolium foi encontrada nas três profundidades amostradas, incrustrando a margem rochosa do tipo arenito, consistindo registro pioneiro desta espécie para a planície de inundação do alto rio Paraná (Ferreira et al. 2011). 
A riqueza de táxons da comunidade de algas perifíticas em $P$. rutifolium foi representada por um total de 137 táxons, distribuídos em nove classes: Bacillariophyceae (52 táxons), Cyanophyceae (48), Zygnemaphyceae (9), Chlorophyceae (9), Euglenophyceae (7), Chrysophyceae (4), Oedogoniophyceae (3), Cryptophyceae (3) e Xanthophyceae (3), A composição taxonômica está apresentada na Tabela 1 .

Maior riqueza das algas perifíticas foi registrada na região profunda ( 88 táxons), reduzindo em direção à região intermediária e subsuperfície (77 e 75 táxons, respectivamente). A maioria das classes estava distribuída uniformemente nas diferentes profundidades (Fig. 2), com exceção de Zygnemaphyceae, classe não representada na região intermediária e com maior valor de riqueza na subsuperfície (sete táxons); Oedogoniophyceae e Cryptophyceae não registradas na subsuperfície; e Xanthophyceae, que ocorreu somente na região mais profunda.

A densidade total de algas perifíticas em $P$. rutifolium correspondeu a $129,7 \times 10^{3}$ ind. $\mathrm{cm}^{-2}$, comportando-se opostamente à riqueza (Fig. 3), pois a maior densidade foi registrada na subsuperfície $\left(66,1 \times 10^{3} \mathrm{ind}, \mathrm{cm}^{-2}\right)$, reduzindo em direção à maior profundidade $\left(37,310^{3} \mathrm{ind}, \mathrm{cm}^{-2}\right.$ na região intermediária e $26,3 \times 10^{3} \mathrm{ind}, \mathrm{cm}^{-2}$ na região profunda), Bacillariophyceae e Cyanophyceae destacaram-se na definição dos valores de densidade total em todas as profundidades. Estas classes apresentaram densidades de 87,6 $\times$ $10^{3}$ ind. $\mathrm{cm}^{-2} \mathrm{e} 39 \times 10^{3}$ ind. $\mathrm{cm}^{-2}$ respectivamente, em contraste com a densidade total das demais classes $\left(3,1 \times 10^{3}\right.$ ind. $\left.\mathrm{cm}^{-2}\right)$. As densidades de Bacillariophyceae e Cyanophyceae diminuíram em direção às regiões mais profundas, enquanto que Chrysophyceae e Euglenophyceae foram mais abundantes em maior profundidade (Fig. 3). Nesta região, as densidades de Xanthophyceae e Zygnemaphyceae também foram mais elevadas.

\section{Discussão}

O regime hidrodinâmico do rio Paraná consiste num fator importante que atua direta ou indiretamente na ocorrênciae desenvolvimento das comunidades aquáticas (Rodrigues \& Bicudo 2001b; Agostinho et al. 2004; Rocha \& Thomaz 2004). Em rios com elevada velocidade de corrente, as macrófitas aquáticas flutuantes são transportadas pela água; enquanto as espécies enraizadas não se desenvolvem sobre o sedimento,
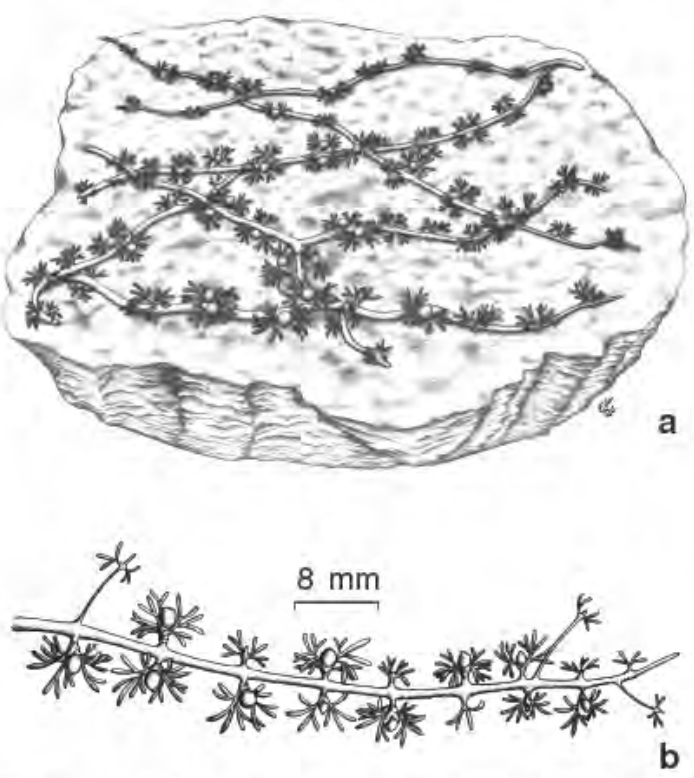

Figura 1 - a-b. Podostemum rutifolium Warm. subsp. rutifolium (F.A.Ferreira 244) - a. hábito; b. detallhe do aspecto geral da planta.

Figure 1 - a-b. Podostemum rutifolium Warm. subsp. rutifolium (F.A.Ferreira 244) - a. habit; b. details of the general appearance of the plant.

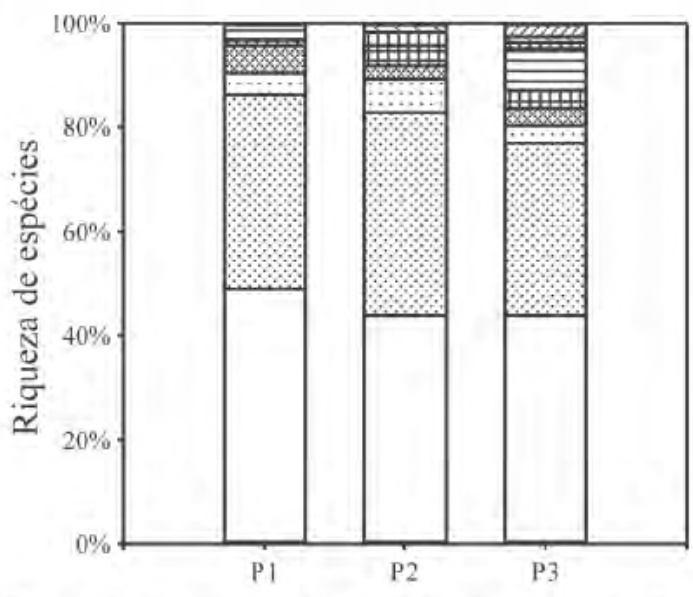

Figura 2 - Contribuição da riqueza de táxons das classes de algas perifiticas em Podostemum rutifolium nas diferentes profundidades no rio Paraná, planície de inundação do alto rio Paraná, em fevereiro de 2009 ( \anthophyceae, 辰Zygnemaphyceae, Chlorophyceae, MIV Cryptophyceae, Chrysophyceae, $\square$ Cyanophyceae, ${ }^{-1 m}$ Oedogoniophyceae, Euglenophyceae,

Bacillariophyceae). $\mathrm{P} 1=0,3, \mathrm{P} 2=1,5 \mathrm{eP} 3=2,3 \mathrm{~m}$. Figure 2 - Species richness of periphytic algae on Podostemum rutifolium at different depths in the Paraná River, at the Paraná River floodplain, in February 2009. 
Tabela 1 - Lista de classes e respectivos táxons de algas perifíticas em Podostemum rutifolium subsp. rutifolium nas três profundidades amostradas $(\mathrm{P} 1=0,3, \mathrm{P} 2=1,5$ e P3 =2,3 m), no rio Paraná, em fevereiro de 2009.

Table 1 - List of classes and respective taxa of the periphytic algae on Podostemum rutifolium subsp. rutifolium in each three sampling points $(\mathrm{P} 1=0,3, \mathrm{P} 2=1,5$ e $\mathrm{P} 3=2,3 \mathrm{~m})$, in the Paraná River, in February 2009.

\section{Táxon}

\section{CYANOPHYCEAE}

Aphanocapsa incerta (Lemmermann) Cronberg \& Komárek Aphanocapsa parasitica (Kützing) Komárek \& Anagnostidis Aphanothece microscopica Nägeli

Borzia trilocularis Cohn ex Gomont

Calothrix fusca (Kützing) Bornet \& Flahault

Chamaesiphon investiens Skuja

Chroococcus dispersus (Keissler) Lemmermann

Chroococcus limneticus Lemmermann

Chroococcus minimus (Keissler) Lemmermann

Chroococcus minor (Kützing) Nägeli

Coelomoron sp.

Cyanostylon microcystoides Geitler

Geitleribactron periphyticum Komárek

Geitlerinema sp.

Gloeocapsa gelatinosa (Meneghini) Kützing

Gloeotrichia echinulata (Smith) Richter

Heteroleibleinia pusilla (Rabenhorst) Compère

Homeothrix stagnalis (Hansgirg) Komárek \& Kováèik

Leibleinia epiphytica (Hieronymus) Anagnostidis \& Komárek

Leptolyngbya foveolarum (Rabenhorst ex Gomont) Anagnostidis \& Komárek

Leptolyngbya lagerheimii (Gomont) Anagnostidis \& Komárek

Leptolyngbya perelegans (Lemmermann) Anagnostidis \& Komárek

Leptolyngbya subtilis Anagnostidis \& Komárek

Leptolyngbya tenuis (Gomont) Anagnostidis \& Komárek

Leptolyngbya sp.

Limnothrix mirabilis (Böcher) Anagnostidis

Lyngbya fragilis (Gomont) Compère

Lyngbya martensiana Meneghini ex Gomont

Lyngbya sp.

Microcystis cf. panniformis Komárek et al.

Oscillatoria annae Van Goor

Oscillatoria cf. perornata Skuja

$x$

Oscillatoria princeps Vaucher ex Gomont

Oscillatoria sancta (Kützing) Gomont

Oscillatoria subbrevis Schmidle

Phormidium granulatum (Gardner) Anagnostidis \& Komárek

Phormidium luridum (Kützing) Gomont

Phormidium molle (Kützing) Gomont

Phormidium sp.

Porphyrosiphon cf. versicolor (Gomont) Anagnostidis \& Komárek Pseudoanabaena minima (An) Anagnostidis

$\begin{array}{lll} & \mathrm{X} & \mathrm{x} \\ & \mathrm{X} & \mathrm{x} \\ \mathrm{X} & & \mathrm{x} \\ \mathrm{X} & \mathrm{X} & \mathrm{x} \\ \mathrm{X} & \mathrm{X} & \\ \mathrm{X} & & \mathrm{x} \\ \mathrm{X} & & \\ \mathrm{X} & \mathrm{X} & \mathrm{x} \\ \mathrm{X} & \mathrm{X} & \mathrm{X} \\ & \mathrm{X} & \mathrm{X} \\ & & \end{array}$

$\mathrm{X}$

$\mathrm{X}$

$\mathrm{X}$

$\mathrm{X}$

$\mathrm{X}$

X

$\mathrm{X}$

$\mathrm{X}$

$\mathrm{X}$

$\mathrm{X}$

X

X

X X X




\begin{tabular}{|c|c|c|c|}
\hline Táxon & $\mathbf{P 1}$ & $\mathbf{P 2}$ & $\mathbf{P 3}$ \\
\hline Pseudanabaena mucicola (Naumann \& Huber-Pestalozzi) Bourrelly & $\mathrm{x}$ & $\mathrm{x}$ & $\mathrm{x}$ \\
\hline Schizothrix sp. & & $\mathrm{x}$ & \\
\hline Synecocysthis aquatilis Sauvageau & $\mathrm{x}$ & & \\
\hline Xenococcus minimus Geitler & $\mathrm{x}$ & & \\
\hline Chroococcales não identificada & $\mathrm{x}$ & $\mathrm{x}$ & $\mathrm{x}$ \\
\hline Chroococcales não identificada 2 & & $\mathrm{x}$ & \\
\hline Cyanophyceae filamentosa não identificada & $\mathrm{x}$ & & \\
\hline \multicolumn{4}{|l|}{ BACILLARIOPHYCEAE } \\
\hline Achnanthidium exiguum (Grunow) Czarnecki & & & $\mathrm{x}$ \\
\hline Achnanthes lanceolata (Brébisson in Kützing) Grunow & & & $\mathrm{x}$ \\
\hline Achnanthes rupestoides Hohn & $\mathrm{x}$ & $\mathrm{x}$ & $\mathrm{x}$ \\
\hline Achnanthidium minutissimum (Kützing) Czarnecki & $\mathrm{x}$ & $\mathrm{x}$ & $\mathrm{x}$ \\
\hline Amphipleura lindheimeri Grunow & $\mathrm{x}$ & & \\
\hline Amphora copulata (Kützing) Schoeman \& Archibald & $\mathrm{x}$ & & $\mathrm{x}$ \\
\hline Aulacoseira alpigena (Grunow) Krammer & & $\mathrm{x}$ & $\mathrm{x}$ \\
\hline Aulacoseira ambigua (Grunow) Simonsen & $\mathrm{x}$ & $\mathrm{x}$ & $\mathrm{x}$ \\
\hline Aulacoseira granulata (Ehrenberg) Simonsen & & $\mathrm{x}$ & $\mathrm{x}$ \\
\hline Cocconeis placentula Ehrenberg & $\mathrm{x}$ & $\mathrm{x}$ & $\mathrm{x}$ \\
\hline Craticula sp. & $\mathrm{x}$ & $\mathrm{x}$ & $\mathrm{x}$ \\
\hline Cyclotella meneghiniana Kützing & $\mathrm{x}$ & $\mathrm{x}$ & \\
\hline Cymbella affinis Kützing & $\mathrm{x}$ & $\mathrm{x}$ & $\mathrm{x}$ \\
\hline Diadesmis confervacea (Grunow) Hustedt & & & $\mathrm{x}$ \\
\hline Encyonema mesianum (Cholnoky) D.G. Mann & $\mathrm{x}$ & $\mathrm{x}$ & $\mathrm{x}$ \\
\hline Encynema minutum (Hilse) D.G. Mann & $\mathrm{x}$ & $\mathrm{x}$ & $\mathrm{x}$ \\
\hline Encyonema perpusillum (Cleve) D.G. Mann & $\mathrm{x}$ & $\mathrm{x}$ & $\mathrm{x}$ \\
\hline Encyonema silesiacum (Bleisch) D.G. Mann & $\mathrm{x}$ & $\mathrm{x}$ & $\mathrm{x}$ \\
\hline Eunotia bilunaris (Ehrenberg) Mills & $\mathrm{x}$ & & \\
\hline Eunotia minor (Kützing) Grunow & & & $\mathrm{x}$ \\
\hline Eunotia monodon Ehrenberg & & $\mathrm{x}$ & $\mathrm{x}$ \\
\hline Eunotia pectinalis (Kützing) Rabenhorst & $\mathrm{x}$ & & $\mathrm{x}$ \\
\hline Eunotia praerupta Ehrenberg & $\mathrm{x}$ & & \\
\hline Eunotia sp. & $\mathrm{x}$ & & \\
\hline Eunotia sp. 2 & & & $\mathrm{x}$ \\
\hline Fragilaria capucina Desmazières & $\mathrm{x}$ & $\mathrm{x}$ & $\mathrm{x}$ \\
\hline Fragilaria tenera (W. Smith) Lange-Bertalot & & $\mathrm{x}$ & \\
\hline Frustulia rhomboides (Ehrenberg) De Toni & & & $\mathrm{x}$ \\
\hline Gomphonema affine Kützing & & $\mathrm{x}$ & \\
\hline Gomphonema augur Ehrenberg & & $\mathrm{x}$ & $\mathrm{x}$ \\
\hline Gomphonema brasiliense Grunow & $\mathrm{x}$ & $\mathrm{x}$ & $\mathrm{x}$ \\
\hline Gomphonema clevei Fricke & $\mathrm{x}$ & $\mathrm{x}$ & $\mathrm{x}$ \\
\hline Gomphonema gracile Ehrenberg emend. Van Heurck & $\mathrm{x}$ & $\mathrm{x}$ & $\mathrm{x}$ \\
\hline Gomphonema parvulum Kützing & $\mathrm{x}$ & $\mathrm{x}$ & $\mathrm{x}$ \\
\hline Gomphonema truncatum Ehrenberg & $\mathrm{x}$ & $\mathrm{x}$ & $\mathrm{x}$ \\
\hline Gyrosigma scalproides (Rabenhorst) Cleve & $\mathrm{x}$ & & $\mathrm{x}$ \\
\hline Melosira varians C.A. Agardh & $\mathrm{x}$ & $\mathrm{x}$ & $\mathrm{x}$ \\
\hline Navicula cf. capitatoradiata Germain & $\mathrm{x}$ & & \\
\hline
\end{tabular}


Navicula constans Hustedt

Navicula cryptotenella Lange-Bertalot

Navicula schroeteri Meister

Navicula viridula (Kützing) Ehrenberg

Navicula sp.

$\mathrm{X}$

$\mathrm{X}$

Neidium cf. catarinense (Krasske) Lange-Bertalot

Nitzschia amphibia Grunow

Nitzschia ignorata Krasske

Nitzschia linearis W. Smith

Nitzschia palea (Kützing) W. Smith

Pinnularia cf. gibba Ehrenberg

Sellaphora pupula (Kützing) Mereschkowsky

Synedra goulardii Brébisson

Terpsinoe musica Ehrenberg

Ulnaria ulna (Nitzsch) Compère

\section{CRYPTOPHYCEAE}

Cryptomonas sp.

Cryptomonas sp. 2

\section{CHLOROPHYCEAE}

\section{Cladophora sp.}

Coenocystis asymmetrica Komárek

Desmodesmus brasiliensis (Bohlin) Hegewald

$\mathrm{X}$

Eutetramorus fottii (Hindák) Komárek

Gloeocystis vesiculosa Nägeli

Uronema sp.

Chlorophyceae colonial cocóide não identificada

Chlorophyceae colonial cocóide não identificada 2

\section{OEDOGONIOPHYCEAE}

Oedogonium sp.

Oedogonium sp. 2

Oedogonium sp. 3

\section{ZYGNEMAPHYCEAE}

Closterium lunula Nitzsch ex Ralfs

Closterium tumidum Johnson

Cosmarium abbreviatum Raciborski

X

Cosmarium galeritum Nordstedt

$\mathrm{x}$

Cosmarium minimum $W$. West \& G. S. West

Cosmarium pseudopyramidatum Lundell

Cosmarium sp.

\section{EUGLENOPHYCEAE}

Phacus agilis Skuja 
Trachelomonas conica Playfair

Trachelomonas hispida (Perty) Stein

Trachelomonas robusta (Swirenko) Deflandre

$\mathrm{x}$

$\mathrm{x}$

Trachelomonas verrucosa Stockes

Trachelomonas volvocinopsis Swirenko

$\begin{array}{lll} & & \mathrm{X} \\ \mathrm{X} & \mathrm{X}\end{array}$

\section{CHRYSOPHYCEAE}

Mallomonas sp.

Mallomonas sp. 2

Mallomonas sp. 3

Mallomonas sp. 4

$\begin{array}{ccc} & & \mathrm{X} \\ & \mathrm{X} & \\ \mathrm{X} & \mathrm{X} & \mathrm{X} \\ & \mathrm{X} & \mathrm{X}\end{array}$

\section{XANTHOPHYCEAE}

Characiopsis aquinolaris Skuja

que se torna instável para sua fixação (Camargo et al. 2003). Já $P$. rutifolium apresenta hábito incrustante em rochas e sedimentos e é extremamente adaptada ao desenvolvimento sob as condições presentes nos ambientes lóticos (Cook 1990; Philbrick et al. 2010). Segundo Philbrick \& Novelo (2004) existe indicação da ocorrência dessa espécie para os rios do Paraguai, Argentina (Corrientes e Missões), Uruguai (rio Uruguai) e Brasil (Rio Grande do Sul, Santa Catarina, Paraná, São Paulo e Rio de Janeiro). Para o Paraná, existem registros para o rio Paraná (próximo a Guaíra) e Parque Nacional do Iguaçu (rio Iguaçu).

A ocorrência desta planta implica não somente na diversidade da comunidade de macrófitas aquáticas, mas também na biodiversidade como um todo (Thomaz et al. 2004), como verificada no presente estudo, para as algas perifíticas. Ao fornecer substrato colonizável ao desenvolvimento do perifíton, a característica riacófila de $P$. rutifolium restringe ecologicamente ambas as comunidades. Isto porque o desenvolvimento e a fisiologia do perifíton estão intrinsecamente relacionados à dinâmica estrutural e funcional do substrato vivo o qual esta comunidade encontra-se aderida (Rodrigues et al. 2003).

Muitos fatores influenciam a estrutura e dinâmica das algas perifíticas em ambientes lóticos, incluindo nutrientes, luz, temperatura, velocidade de corrente, substrato e herbivoria (Goldsborough \& Robinson 1996; Jones et al. 2000). As algas perifíticas no rio Paraná mostraram-se estruturadas conforme o substrato representado por $P$. rutifolium, frente às características hidrodinâmicas e limnológicas do ambiente, decorrente do hábito riacófilo desta macrófita. Tais características estiveram representadas pela corrente de água e profundidade. Em ambientes lóticos, as perturbações causadas pelas inundações afetam a biota aquática deste ambiente diretamente e atuam como o principal fator na estruturação e disponibilidade de habitat (Webb et al. 2006).

O rio Paraná é caracterizado por elevados valores de transparência da água e baixa concentração de nutrientes, principalmente fósforo (Rodrigues \& Bicudo 2001a; Rocha \& Thomaz 2004; Roberto et al. 2009; Thomaz et al. 2009), além de apresentar maior velocidade de corrente que lagoas e ressacos. Estes fatores atuam como perturbações ao desenvolvimento da comunidade de algas perifíticas, possibilitando a presença e permanência apenas de grupos capazes de tolerar condições estressantes. No presente estudo, esse fato foi corroborado pela abundância e dominância dos grupos Bacillariophyceae e Cyanophyceae, algas extremamente tolerantes às condições ambientais. Principalmente, na região superficial, onde a corrente de água foi mais intensa.

A classe Bacillariophyceae, representada pelas diatomáceas, possui a habilidade de se desenvolver em baixas concentrações de fósforo, porque requerem um menor suprimento deste nutriente para o seu desenvolvimento (Winter \& Duthie 2000; Rodrigues \& Bicudo 2001b). Além 

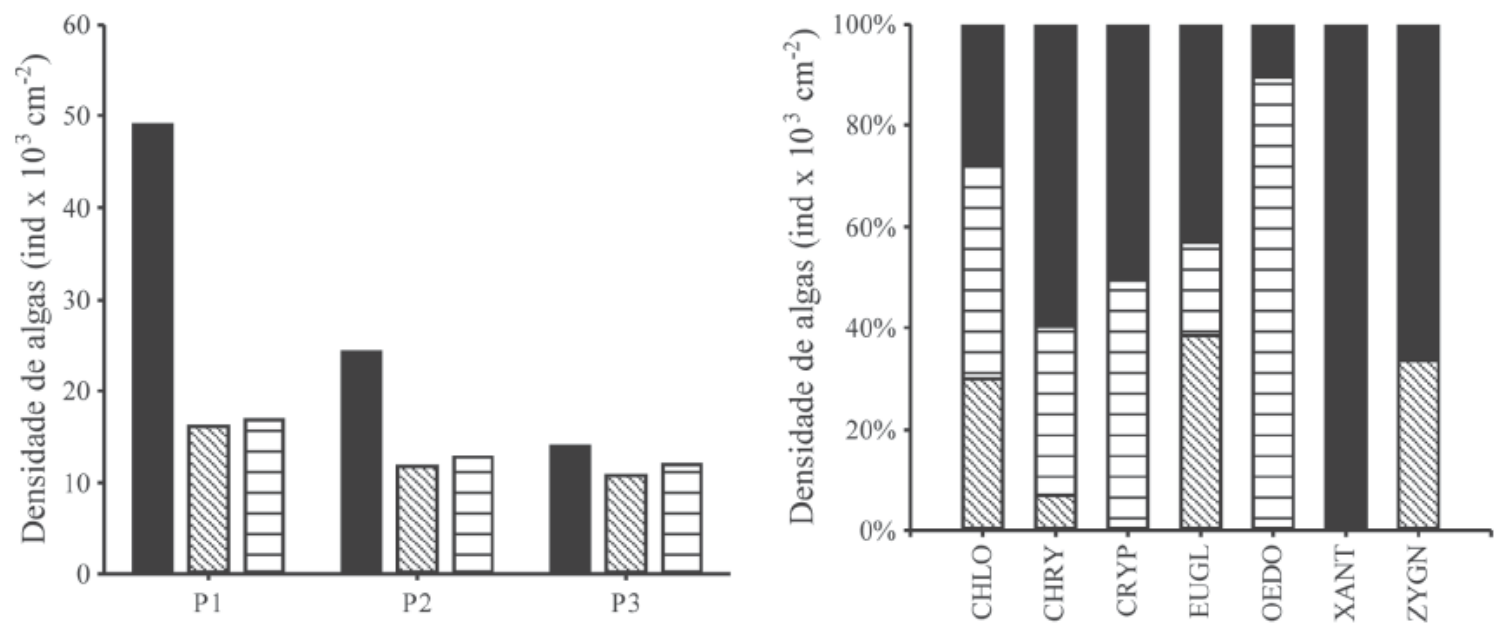

Figura 3 - a. Densidade das principais classes de algas perifiticas sobre Podostemum rutifolium subsp. rutifolium nas diferentes profundidades no rio Paraná, planície de inundação do alto rio Paraná, em fevereiro de 2009

Bacillariophyceae, $\cong$ Cyanophyceae, $\rightleftharpoons$ demais classes). b. Contribuição das densidades das demais classes de algas perifíticas em $P$. rutifolium nas diferentes profundidades no rio Paraná, planície de inundação do alto rio Paraná, em fevereiro de 2009 (CHLO = Chlorophyceae, CHRY = Chrysophyceae, CRYP = Cryptophyceae, EUGL = Euglenophyceae, OEDO = Oedogoniophyceae, XANT = Xanthophyceae, ZYGN = Zygnemaphyceae, 르 P1,

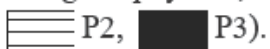

Figure 3 - a. Main classes density of periphytic algae on Podostemum rutifolium subsp. rutifolium at different depths in the Paraná River, at the Paraná River floodplain, in February 2009 (Bacillariophyceae, Cyanophyceae, other classes). b. Density contribution of other periphytic algae classes on P. rutifolium at different depths in the Paraná River, at the Paraná River floodplain, in February 2009.

disso, as diatomáceas apresentam baixa exigência de energia para crescimento quando comparada às algas não-silicosas, em razão da utilização de silicato em suas células (Rodrigues \& Bicudo 2001b) presente em maiores concentrações nos ambientes lóticos da planície em comparação aos lênticos (Rodrigues \& Bicudo 2001a), especialmente o rio Paraná. Por se tratar, portanto, de indivíduos com rápida capacidade de colonização e atuarem como competidores eficientes (Stevenson 1996; Biggs 1996), e por apresentar menores requisitos de nicho, suportando condições desfavoráveis, este grupo dominou perante os demais.

Ainda, as diatomáceas são favorecidas em ambientes aquáticos com regime hidrodinâmico de alta corrente e vazão, porque são capazes de desenvolver estruturas adaptativas de fixação no substrato (Biggs 1996), como pedúnculos mucilaginosos, produção de matrizes de mucilagem, e colônias em forma de estrela ou ramos, fixas pela base (Hoagland et al. 1982; Murakami et al. 2009). Estas estruturas propiciam grande vantagem a estas algas para sua permanência na comunidade perifítica e resistência frente às perturbações. Acredita-se por todas estas razões que na comunidade perifítica em $P$. rutifolium, tanto em termos de riqueza quanto densidade, Bacillariophyceae foi a classe responsável pela excepcional dominância, juntamente com Cyanophyceae (cianobactérias). Resultados semelhantes foram registrados para a comunidade perifítica sobre Eichhornia azurea Kunth presente nos distintos ambientes da planície de inundação do alto rio Paraná (Rodrigues \& Bicudo 2001b; Fonseca \& Rodrigues 2005a, Algarte et al. 2006).

As cianobactérias (Cyanophyceae) são consideradas organismos cosmopolitas e colonizadores amplamente oportunistas (Huszar et al. 2000; Fonseca \& Rodrigues 2005a,b). Apresentam características relacionadas à atividade fotossintética que permitem absorção mais eficiente de luz e assim requerem menor quantidade de energia para o seu desenvolvimento (Chorus \& Bartram 1999), além da capacidade de armazenamento do fósforo na forma de grãos citoplasmáticos (Sant'anna et al. 2006). Menor requerimento de fósforo externo favorece este grupo em ambientes onde este recurso é limitante, como no rio Paraná. Conjuntamente ao elevado requerimento de nitrogênio (Chorus \& Bartram 1999; Fonseca \& Rodrigues 2005a), nutriente disponível 
em altas concentrações no rio Paraná (Rodrigues \& Bicudo 2001a), estas condições habilitam as cianobactérias como melhores competidoras em relação aos outros grupos algais. Estes fatores podem ter propiciado o desenvolvimento deste grupo de forma predominante na comunidade perifítica de $P$. rutifolium.

Os representantes das Zygnemaphyceae são abundantes em águas colonizadas amplamente por macrófitas (Coesel 1982, 1996; Felisberto \& Rodrigues 2005; Murakami et al. 2009) e estão presentes tanto entre a vegetação aquática, quanto aderidos ao substrato. Apesar da baixa riqueza de Zygnemaphyceae e Chlorophyceace no presente estudo, a elevação dos valores destas classes na região mais profunda podem ser coincidentes com o aumento das macrófitas submersas no rio Paraná. De acordo com Thomaz et al. (2004, 2009), a operação das barragens a montante, com consequente aumento da transparência da água e redução das concentrações de fósforo, têm sido responsável pelo aumento das formas submersas ao longo do rio. Por sua vez, a elevação da diversidade de macrófitas, que propiciam um substrato colonizável para a comunidade perifítica, contribui consideravelmente para o aumento da riqueza de algas perifíticas, principalmente Zygnemaphyceae (Murakami et al. 2009). Sugerese, desta forma, a influência do intercâmbio de algas perifíticas das macrófitas submersas para demais substratos, como $P$. rutifolium.

Outro fato relevante observado são os maiores valores na densidade e riqueza das classes menos representativas em maiores profundidades, conjuntamente ao decréscimo da riqueza e densidade de Bacillariophyceae e Cyanophyceae (grupos dominantes) nesta região. Em outros estudos, este padrão inverso de riqueza e densidade também foi observado para a comunidade de algas perifíticas nos distintos ambientes da planície do rio Paraná (Rodrigues \& Bicudo 2001b; Fonseca \& Rodrigues 2005a; Algarte et al. 2006, 2009; Murakami et al. 2009). No presente estudo, este fato pode ser explicado pela diminuição da velocidade da água com o aumento da profundidade. Tal fato propicia o desenvolvimento de uma maior riqueza de táxons pelo menor nível de perturbação e pela influência das macrófitas submersas, o que permite o desenvolvimento de formas mais frouxamente aderidas como Zygnemaphyceae e Chlorophyceae. Todavia, uma análise mais detalhada da comunidade poderá evidenciar os fatores físicos, químicos e biológicos que contribuem para as diferenças que ocorrem nas diferentes profundidades.

Os impactos da fauna herbívora sobre as algas perifíticas em planícies de inundação podem provocar alterações na estrutura das comunidades perifíticas. Relações dos efeitos dos herbívoros e a natureza do substrato também contribuem para afetar a estrutura algal no perifíton (Goldsborough \& Robinson 1996; Jones et al. 2000; Mormul et al. 2010). As Podostemaceae são reconhecidamente caracterizadas como importantes fontes de alimento para diversos herbívoros aquáticos (Vilella et al. 2002). Além disso, as algas perifíticas, por sua vez, podem contribuir como fonte de nutrientes para a fauna herbívora, influenciando a cadeia dos consumidores e a exportação de matéria orgânica e energia (Campeau et al. 1994; Fonseca \& Rodrigues 2005a). Ainda, a cobertura pelo perifíton proporciona vantagens ao substrato, ao evitar a herbivoria direta à planta hospedeira (Eminson \& Moss 1980; Jones et al. 2000). Sugere-se, a partir do presente estudo, a importância tanto da presença de $P$. rutifolium como da rica comunidade perifítica sustentada por esta planta como importantes elos na cadeia alimentar aquática do rio Paraná. Atenta-se para o desenvolvimento de futuros estudos acerca das interações tróficas envolvendo esta macrófita e o perifíton dentre outras comunidades aquáticas neste ambiente.

A despeito das considerações expostas, verifica-se, de forma geral, a importância do substrato na estruturação das comunidades algais. No presente estudo, a presença do substrato constituído pela macrófita $P$. rutifolium influenciou na estruturação das algas perifíticas, juntamente aos fatores limnológicos característicos do regime hidrodinâmico do rio Paraná. Esta observação é de grande relevância na conservação destes ambientes, porque variações na composição das comunidades das macrófitas podem determinar, por sua vez, os padrões de biodiversidade de outras comunidades aquáticas (Thomaz et al. 2004). Além disso, é importante monitorar a dinâmica da comunidade perifítica, porque esta atua como elo na cadeia alimentar aquática, o que reflete em outros grupos ecológicos no sistema (Murakami et al. 2009). Sugere-se, portanto, que $P$. rutifolium seja considerada uma espécie estruturadora chave, segundo o conceito de "keystone structure" (Mills et al. 1993), uma vez que constitui o único substrato vegetal, de hábito incrustrante e riacófila, na área 
amostrada (região litorânea do rio Paraná); e pela qual sua remoção poderia levar ao declínio na densidade e diversidade das demais comunidades associados a ela, enfaticamente, o perifíton. Apesar da disponibilidade de demais substratos disponíveis à comunidade perifítica no rio Paraná como rochas e sedimento, a dinâmica do perifíton sobre um substrato vivo é completamente particular e pode definir a estrutura e composição das algas aderidas.

\section{Agradecimentos}

Os autores agradecem à Coordenação de Aperfeiçoamento de Pessoal de Nível Superior (CAPES) e ao Conselho Nacional de Desenvolvimento Tecnológico e Científico (CNPq) às bolsas de pósgraduação; á Aldaléa Sparada Tavarez pela identificação da espécie de Podos temaceae e Eduardo Ribeiro da Cunha pela ilustração botânica.

\section{Referências}

Agostinho, A.A.; Gomes, L.C. \& Julio Jr, H.F. 2003. Relações entre macrófitas aquáticas e fauna de peixes. In: Thomaz, S.M. \& Bini, L.M. Ecologia e manejo de macrófitas aquáticas. Eduem, Maringá. Pp. 261-277.

Agostinho, A.A.; Thomaz, S.M. \& Gomes, LC. 2004. Threats for biodiversity in the floodplain of the Upper Paraná River: effects of hydrological regulation by dams. Ecohydrology \& Hydrobiology 4: 255-268.

Agostinho, A.A.; Gomes, L.C. \& Pelicice, F.M. 2007. Ecologia e manejo de recurso pesqueiro em reservatórios do Brasil. Eduem, Maringá. 501p.

Algarte, V.M.; Moresco, C. \& Rodrigues, L. 2006. Algas do perifiton de distintos ambientes na planície de inundação do alto rio Paraná. Acta Scientiarum (Biological Sciences) 28: 243-251.

Algarte, V.M.; Siqueira, N.S.; Murakami, E.A. \& Rodrigues, L. 2009. Effects of hydrological regime and connectivity on the interannual variation in taxonomic similarity of periphytic algae. Brazilian Journal of Biology 69: 609-616.

Anagnostidis, K. \& Komárek, J. 1988. Modern approach to the classification system of Cyanophytes 3 Oscillatoriales. Archiv Hydrobiology Supplement 80: 327-472.

Bicudo, C.E.M. \& Menezes, M. 2006. Gênero de algas de águas continentais do Brasil. Chave para identificação e descrição. Rima, São Carlos. 502p.

Bicudo, C.M. 1990. Considerações sobre metodologias de contagem de algas do perifiton. Acta Limnologica Brasiliensia 3: 459-475.

Biggs, B.J.F. 1996. Patterns in benthic algal of streams. In: Stevenson, R.J.; Bothwell, M.L. \& Lowe, R.L.
Algal ecology: freshwater benthic ecosystems. Academic Press, New York. Pp. 31-56.

Biolo, S. \& Rodrigues, L. 2010. New records of Xanthophyceae and Euglenophyceae in the periphytic algal community from a neotropical river floodplain, Brazil. Algological Studies 135: 61-81.

Biolo, S. \& Rodrigues, L. 2011. Composição de algas perifíticas (exceto Bacillariophyceae) em distintos substratos naturais de um ambiente semilótico, planície de inundação do Alto Rio Paraná, Brasil. Revista Brasileira de Botânica 34: 307-319.

Camargo, A.F.M.; Pezzato, M.M. \& Henry-Silva, G.G. 2003. Fatores limitantes à produção primária de macrófitas aquáticas. In: Thomaz, S.M. \& Bini, L.M. Ecologia e manejo de macrófitas aquáticas. Eduem, Maringá. Pp. 59-83.

Campeau, S.; Murkin, H.R. \& Titman, R.D. 1994. Relative importance of algae and emergent plant litter to freshwater marsh invertebrates. Canadian Journal of Fisheries and Aquatic Sciences 51: 681-692.

Chorus, I. \& Bartram, J. 1999. Toxic cyanobacteria in water: a guide to their public health consequences, monitoring and management. E \& FN SPON, London. 416p.

Coesel, P.F.M. 1982. Structural characteristics and adaptations of desmid communities. Journal of Ecology 70: 163-177.

Coesel, P.F.M. 1996. Biogeography of desmids. Hydrobiologia 336: 41-53.

Cook, C.D.K. 1990. Aquatic plant book. SPB Academic Publishing, The Hague. 228p.

Eminson, D.F. \& Moss, B. 1980. The composition and ecology of periphyton communities in freshwaters. I. The influence of host type and external environment on community composition. European Journal of Phycology 15: 429-446.

Estes, J.A.; Terborgh, J.; Brashares, J.S.; Power, M.E.; Berger, J.; Bond, W.J.; Carpenter, S.R.; Essington, T.E.; Holt, R.D.; Jackson, J.B.C.; Marquis, R.J.; Oksanen, L.; Oksanen, T.; Paine, R.T.; Pikitch, E.K.; Ripple, W.J.; Sandin, S.A.; Scheffer, M.; Schoener, T.W.; Shurin, J.B.; Sinclair, A.R.E.; Soulé, M.E.; Virtanen, R. \& Wardle, D.A. 2011. Trophic downgrading of planet Earth. Science 333: 301-306.

Esteves, F.A. 1998. Fundamentos de limnologia. 2ed. Interciência, Rio de Janeiro. 602p.

Felisberto, S.A. \& Rodrigues, L. 2005. Influência do gradiente longitudinal (rio-barragem) na similaridade das comunidades de desmídias perifíticas. Revista Brasileira de Botânica 28: 241-254.

Ferreira, F.A.; Mormul, R.P.; Thomaz, S.M.; Pott, A. \& Pott, V.J. 2011. Macrophytes in the Upper Paraná River floodplain: checklist and comparison with other large South American wetlands. Revista de Biologia Tropical 59: 541-556.

Fonseca, I.A. \& Rodrigues, L. 2005a. Comunidade de algas perifíticas em distintos ambientes da planície 
de inundação do alto rio Paraná. Acta Scientiarum (Biological Sciences) 27: 21-28.

Fonseca, I.A. \& Rodrigues, L. 2005b. Cianobactérias perifíticas em dois ambientes lênticos da planície de inundação do alto Rio Paraná, PR, Brasil. Revista Brasileira de Botânica 28: 821-834.

Förster, K. 1969. Amazonische Desmidiaceen. 1. Teil. Areal Santarém. Amazoniana 11: 5-116.

Goldsborough, G. \& Robinson, G.G.C. 1996. Pattern in Wetlands. In: Stevenson, R.J.; Bothwell, M.L.\& Lowe, R.L. Algal ecology: freshwater benthic ecosystems. Academic Press, New York. Pp. 78-119.

Hoagland, K.D.; Roemer, S.C. \& Rosowski, J.R. 1982. Colonization and community structure of two periphyton assemblages, with emphasis on the diatoms (Bacillariophyceae). American Journal of Botany 69: 188-213.

Huszar, V.L.M.; Silva, L.H S.; Marinho, M.; Domingos, P. \& Sant'anna C.L. 2000. Cyanoprokaryote assemblages in eight productive tropical Brazilian waters. Hydrobiologia 424: 67-77.

Irgang, B.E.; Gastal Jr.; C.V.S.; Philbrick, C.T. \& Novelo, A. 2003. A ocorrência inédita de uma Podostemaceae nas costas de uma laguna (Laguna dos Patos) no Rio Grande do Sul, Brasil. Caderno de Pesquisa (Série Biologia) 15: 7-12.

Jones, J.I.; Moss, B.; Eaton, J.W. \& Young, J.O. 2000. Do submerged aquatic plants influence periphyton community composition for the benefit of invertebrate mutualists? Freshwater Biology 43: 591-604.

Komárek, J. \& Anagnostidis, K. 1986. Modern approach to the classification system of Cyanophytes 2: Chroococcales. Archiv Hydrobiology Supplement 73: $157-226$.

Komárek, J. \& Anagnostidis, K. 1999. Cyanoprokaryota I Teil Chroococcales. In: Ettl, H.; Gerloff, J.; Heyning, H. \& Mollenhauer, D. (eds.). Süsswasser flora von Mitteleuropa. Gustav Fischer Verlag, Stuttgart. 548p.

Komárek, J. \& Anagnostidis, K. 2005. Cyanoprokaryota II. Teil 2nd Part: Oscillatoriales. In: Büdel, B.; Krienitz, L.; Gärtner, G. \& Schagerl, M. (eds.). Süsswasser flora von Mitteleuropa. Elsevier/ Spektrum, Heidelberg. 759p.

Komárek, J. \& Fott, B. 1983. Chlorophyceae Chlorococcales. In: Huber-Pestalozzi, G. (ed.). Das Phytoplankton des Süsswassers. Systematik und Biologie. Vol. 16. E. Schweizerbart'sche Verlagsbuchhandlung, Stuttgart. 1044p.

Krammer, K. \& Lange-Bertalot, H. 1986. Bacillariophyceae: Naviculaceae. In: Ettl, H.; Gerloff, J.; Heyning, H. \& Mollenhauer, D. (eds.). Süsswasser flora von Mitteleuropa. Vol. 2, part. 1. Gustav Fischer Verlag, Stuttgart. Pp. 1-876.

Krammer, K. 1988. Bacillariophyceae: Bacillariaceae, Epithemiaceae, Surirellaceae. In: Ettl, H.; Gerloff, J.; Heyning, H. \& Mollenhauer, D. (eds.).
Süsswasser flora von Mitteleuropa. Vol. 2, part. 2. Gustav Fischer, Stuttgart. Pp. 1-596.

Krammer, K. \& Lange-Bertalot, H. 1991a. Bacillariophyceae. Achnanthaceae. Kritische Ergänzungen zu Navicula (Linolatae) und Gomphonema. In: Ettl, H.; Gerloff, J.; Heyning, H. \& Mollenhauer, D. (eds.). Süßwasserflora von Mitteleuropa. Gustav Fisher, Stuttgart. Pp. 1-437.

Krammer, K. \& Lange-Bertalot, H. $1991 \mathrm{~b}$. Bacillariophyceae: Centrales, Fragilariaceae, Eunotiaceae. In: Ettl, H.; Gerloff, J.; Heyning, H. \& Mollenhauer, D. (eds.). Süßwasserflora von Mitteleuropa. Gustav Fisher, Stuttgart. Pp. 1-576.

Leandrini, J. \& Rodrigues, L. 2008. Variação temporal da biomassa perifítica em ambientes semilóticos da planície de inundação do alto Rio Paraná. Acta Limnologica Brasiliensia 20: 21-28.

Leandrini, J.; Fonseca, I.A. \& Rodrigues, L. 2008. Variation in algal periphyton biomass in the upper Paraná River floodplain, Brazil. Brazilian Journal of Biology 68: 14-37.

Lopes, C.A.; Benedito-Cecilio, E. \& Martinelli, L.A. 2007. Variability in the carbon isotope signature of Prochilodus lineatus (Prochilodontidae, Characiformes) a bottom-feeding fish of the Neotropical region. Journal of Fish Biology 70: 1649-1659.

Maltchik, L. \& Callisto, M. 2004. The use of rapid assessment approach to discuss ecological theories in wetland systems, southern Brazil. Interciencia 29: 219-223.

Mills, L.S.; Soulé, M.E. \& Doak, D.F. 1993. The keystonespecies concept in ecology and conservation. BioScience 43: 219-224.

Mormul, R.P.; Thomaz, S.M.; Silveira, M.J. \& Rodrigues, L. 2010. Epiphyton or macrophyte: Which primary producer attracts the snail Hebetancylus moricandi? American Malacological Bulletin 28: 127-133.

Murakami, E.A.; Bicudo, D.C. \& Rodrigues, L. 2009. Periphytic algae of the Garças Lake, Upper Paraná River floodplain: comparing the years 1994 and 2004. Brazilian Journal of Biology 69: 459-468.

Murphy, K. J.; Dickinson, G.; Thomaz, S. M.; Bini, L. M.; Dick, K.; Greaves, K.; Kennedy, M.P.; Livingstone, S.; McFerran, H.; Milne, J.M.; Oldroyd, J. \& Wingfield, R.A. 2003. Aquatic plant communities and predictors of diversity in a subtropical river floodplain: the upper Rio Paraná, Brazil. Aquatic Botany 77: 256-276.

Neiff, J.J. 1990. Ideas para la interpretación ecológica del Paraná. Interciencia 15: 424-441.

Paine, R.T. 1969. A note on trophic complexity and community stability. The American Naturalist 103: 91-93.

Prescott, G.W. 1982. Algae of the western great lakes area. Otto Koeltz Science Publishers, Koenigstein. 977p.

Prescott, G.W.; Croasdale, H.T. \& Vinyard, W.C. 1981. A synopsis of North American desmids. Part II. Desmidiaceae: Placodermae. Section 3. In: Prescott, 
G.W. (ed.). Desmidiales. University Nebraska Press, Lincoln. 720p.

Philbrick, C.T. \& Novelo, A.R. 2004. Monograph of Podostemum (Podostemaceae). Systematic Botany Monographs 70: 1-106

Philbrick, C.T.; Bove, C.P. \& Stevens, H.I. 2010. Endemism in neotropical Podostemaceae. Annals of the Missouri Botanical Garden 97: 425-456.

Roberto, M.C.; Santana, N.F. \& Thomaz, S.M. 2009. Limnology in the Upper Paraná River floodplain: large-scale spatial and temporal patterns, and the influence of reservoirs. Brazilian Journal of Biology 69: 717-725.

Rocha, R.R.A. \& Thomaz, S.M. 2004. Variação temporal de fatores limnológicos em ambientes da planície de inundação do alto rio Paraná (PR/MS - Brasil). Acta Scientiarum (Biological Sciences) 26: 261-271.

Rodrigues, L. \& Bicudo, D.C. 2001a. Limnological characteristics comparison in three systems with different hydrodynamic regime in the upper Paraná river floodplain. Acta Limnologica Brasiliensia 13: 39-49.

Rodrigues, L. \& Bicudo, D.C. 2001b. Similarity among periphyton algal communities in a lentic-lotic gradient of the upper Paraná river floodplain, Brazil. Revista Brasileira de Botânica 24: 235-248.

Rodrigues, L.; Bicudo, D.C. \& Moschini-Carlos, V. 2003. $\mathrm{O}$ papel do perifíton em áreas alagáveis e nos diagnósticos ambientais. In: Thomaz, S.M. \& Bini, M.L. (eds.). Ecologia e manejo de macrófitas aquáticas. Eduem, Maringá. Pp. 211-230.

Rodrigues, L.; Zanon, J.E.; Biolo, S. \& Carapunarla, L. 2008. A planície alagável do rio Paraná: estruturas e processos ambientais - Perifíton. Programa de Pesquisas Ecológicas de Longa Duração - PELD/CNPq. Relatório técnico-científico das atividades de 2008. Disponível em <http://www.peld.uem.br/Relat2008/ pdf/Capitulo04.pdf>.

Ros, J. 1979. Práticas de ecologia. Omega, Barcelona. 181p.

Sant'anna, C.L.; Azevedo, M.T.P.; Agujaro, L.F.; Carvalho, M.C.; Carvalho, L.R. \& Souza, R.C.R. 2006. Identificação e contagem de cianobactérias planctônicas de águas continentais brasileiras. Interciência, Rio de Janeiro. 58p.

Stevenson, R.J. 1996. An introduction to algal ecology in freshwater benthic habitats. In: Stevenson, R.J., Bothwell, M.L. \& Lowe, R.L. (eds.). Algal ecology: freshwater benthic ecosystems. Academic Press, New York. Pp. 3-30.
Tews, L.; Brose, U.; Grimm, V.; Tielbörger, K.; Wichmann, M.C.; Schwager, M. \& Jeltsch, F. 2004. Animal species diversity driven by habitat heterogeneity/diversity: the importance of keystone structures. Journal of Biogeography 31: 79-92.

Thomaz, S.M.; Bini, L.M. \& Bozeli, R.L. 2007. Floods increase similarity among aquatic habitats in riverfloodplain systems. Hydrobiologia 579: 1-13.

Thomaz, S.M.; Bini, L.M.; Pagioro, T.A.; Murphy, K.J.; Santos, A.M. \& Souza, D.C. 2004. Aquatic macrophytes: diversity, biomass and decompostition. In: Thomaz, S.M.; Agostinho, A.A. \& Hahn, N.S. (eds.). The upper Paraná River and its floodplain: physical aspects, ecology and conservation. Backhuys Publishers, Leiden. Pp. 331-352.

Thomaz, S.M.; Carvalho, P.; Padial, A.A. \& Kobayashi, J.T. 2009. Temporal and spatial patterns of aquatic macrophyte diversity in the Upper Paraná River floodplain. Brazilian Journal of Biology 69: 617-625.

Thomaz, S.M.; Roberto, M.C. \& Bini, L.M. 1997. Caracterização limnológica dos ambientes aquáticos e influência dos ambientes aquáticos e influência dos níveis fluviométricos. In: Vazzoler, A.E.A.M.; Agostinho, A.A. \& Hanh, N.S. (eds.). A planície de inundação do alto rio Paraná. Eduem, Maringá. Pp. 73-102.

Utermöhl, H. 1958. Zur vervollkommung der quantitative phytoplankton-methodik. Mitteilungen Internationale Vereinigung für Theoretische und Angewandte Limnologie 9: 1-38.

Vilella, F.S.; Becker, F.G. \& Hartz, S.M. 2002. Diet of Astyanax species (Teleostei, Characidae) in an Atlantic forest river in Southern Brazil. Brazilian Archives of Biology and Technology 45: 223-232.

Webb, J.A.; Downes, J.A.; Lake, P.S. \& Glaister, A. 2006. Quantifying abrasion of stable substrata in streams: a new disturbance index for epilithic biota. Hydrobiologia 559: 443-453.

Wetzel, R.G. 2001. Limnology: lake and river ecossistems. Academic Press, San Diego. 10066p.

Winter, J.G. \& Duthie, H.C. 2000. Epilithic diatoms as indicators of stream total $\mathrm{N}$ and $\mathrm{P}$ concentration. Journal of North American Benthological Society 19: 32-49. 